\title{
Research on Interference Optimization Mechanism of Wireless Routing Signal Transmission Based on Fuzzy Comprehensive Evaluation Method
}

\author{
https://doi.org/10.3991/ijoe.v13i03.6866 \\ Xinchun Wang* \\ North China University of Science and Technology, Tangshan, China \\ 10759697 @qq.com \\ Haishan Zhang \\ North China University of Science and Technology, Tangshan, China \\ 38775617 @qq. com \\ $\mathrm{Mi} \mathrm{Li}$ \\ North China University of Science and Technology, Tangshan, China \\ $994177231 @ q q \cdot c o m$ \\ Ying Li \\ North China University of Science and Technology, Tangshan, China \\ 1965063794 @qq. com
}

\begin{abstract}
Inherently dynamic in nature, wireless sensor network transmits data less efficient and reliable, and thus the conventional routing protocol is inapplicable to the new type of wireless sensor network. Such being the case, this paper first analyzes this problem and proposes a routing interference optimization mechanism FCE based on distributed fuzzy comprehensive evaluation. The fuzzy principle is introduced to the priority calculation of the node. In doing so, the fuzzy linear transformation principle and the maximum membership principle are used to classify the dynamic nature of candidate nodes and to select well-performed candidates from the optimal candidate nodes. In spite of the few number of those candidates, they are qualified to compete for the right of nexthop forwarding, such that the routing interference will become less probable and the data forwarding is rendered more efficient. Finally, through a simulation test, our method is verified effective.
\end{abstract}

Keywords - comprehensive evaluation; signal transmission; interference optimization; forwarding efficiency.

\section{Introduction}

In practical application, wireless sensor networks are frequently exposed to multiple objective factors such as link quality, node cycle and the addition of nodes, and thus endow network topology with the dynamic nature. Because these factors are 
objective, dynamics becomes one of the essential characteristics of wireless sensor network, exerting strong effects on the network data transmission [1-4]. The routing protocol based on traditional routing tables fails to meet the requirements of wireless sensor networks of a large number, mostly due to the incapability to response well to the dynamic changes of network topology [5-6]. To this end, some dynamic routing mechanisms are proposed to replace the traditional method of routing table update, in which the network can be routed through the location information of the nodes without resource consumption. In this way, the routing protocol is applicable to wireless sensor networks of strong dynamic nature. Nevertheless, under such routing conditions, the competition between multiple candidate nodes will have a great impact on the transmission of the network. In some networks where nodes are densely distributed, it is likely that network congestion is caused by node conflict, which significantly affects the transmission of the network and may lead to a large number of packet loss failures [7-10].

In order to solve the above problems, several new routing protocols are proposed, which can prioritize the forwarding area of the nodes. They play certain roles in reducing the number of candidate nodes and buffering the effect of routing interference [11]. However, higher demands arise for these protocols to push through the limitations of performance and serve for unevenly distributed network nodes. An example of the necessity of protocol modification is when the maximum competition response interval is fixed, the probability of interference caused by node competition in the corresponding densely distributed area will increase rapidly, whilst such phenomenon rarely appears in sparsely distributed areas. Therefore, the requirements for network load balance cannot be satisfied, and the network service performance is affected accordingly. As a comparison, CCMR protocol is a good problem-solver, with the sifting mechanism of low interference probability. In this protocol, the system analyzes the correlation between forwarding and candidate nodes, and then orders the priority of candidate nodes at the number range of (0. 1). By sending several rounds of forwarding requests REQ, the CCMR protocol sets limits to the cost range of nodes, and finally selects the candidates that meet the forwarding cost requirements [12]. Actually, the drawback of this solution is the complex computation of network node density parameters. Therefore, it is necessary to continue the research and analysis of competition-induced interference between candidate nodes.

\section{Descriptions and analysis of routing conflicts}

When the information is transmitted through the stateless geography forwarding protocol, the next-hop forwarding nodes can be selected from the competing candidate nodes, whose diagram is shown in Figure 1 [13]. For example, when S node is required to forward data, it first broadcasts RTS frame to other nodes within the area. Those who receive the frame are allowed to compete for the next-hop forwarding and to do calculations according to location information. If the distance between the base station and itself is shorter than that between the S node and itself, the node will identify its priority level according to forwarding distance, and then select the corresponding time slot for CTS transmission. 


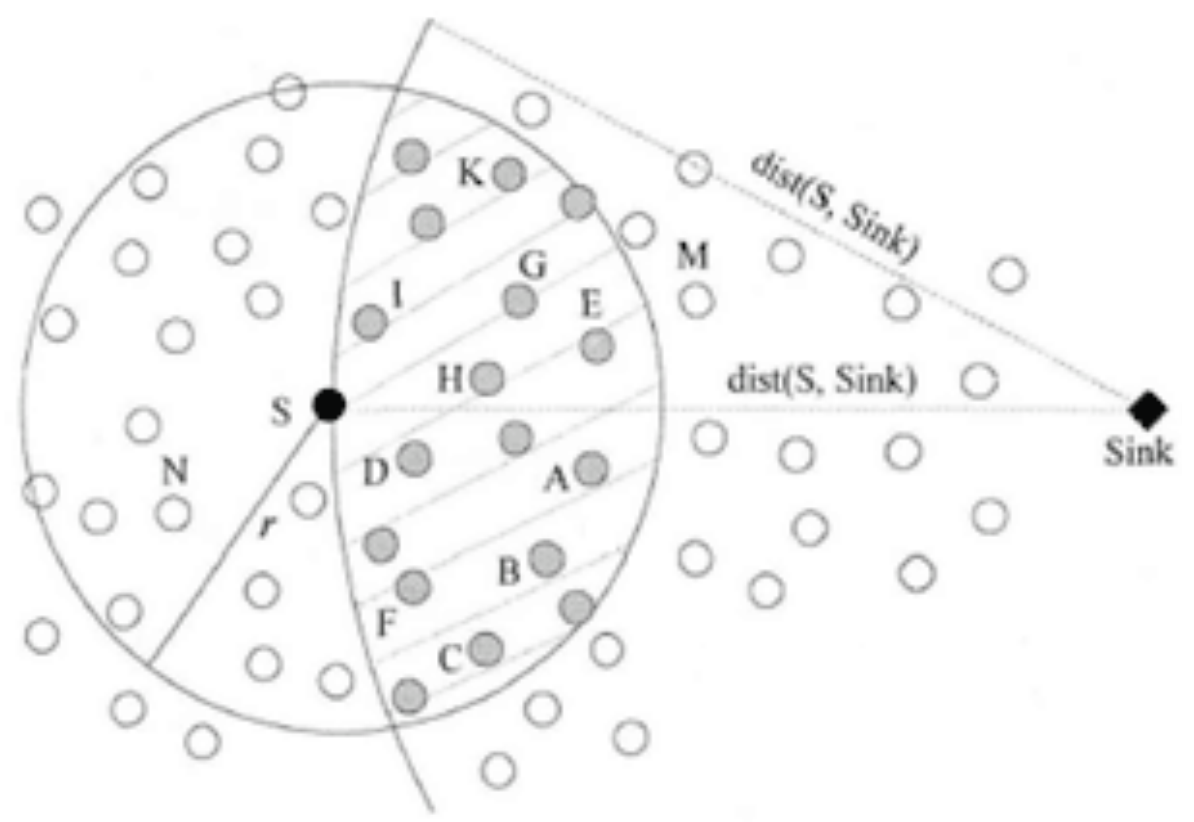

Fig. 1. The diagram of stateless forwarding

During data transmission through the stateless geography forwarding protocol, the order of time slot numbers is mainly related to the priority. The higher the priority level is, the smaller the node's order number is [14-15]. Below is a case analysis. In Figure 2-a, node A has the highest priority level at time slot No.3. At the beginning of the competition, each candidate node is waiting for their exclusive slots. In this case, the candidate nodes with smaller order number are the earliest to send CTS. In this figure, after node A sends information in the No.3 time slot, the rest of the nodes will quit transmitting data upon receiving the forwarding signal, which indicates the competition of channels is completed successfully. Afterwards, node $\mathrm{S}$ will either forward data to node A, or restore itself to the initial state and repeat the above steps until the new round of data transmission is finished with the base station as the termination [16-17].

In the first round of selection, if there are more than one candidate nodes selecting the same time slot, the CTS-competition-induced interference between nodes is inevitable. Taking Figure 2-b as an example, time slot No.4 is the common choice of node A and node $\mathrm{E}$, which means that the pair of nodes will have traffic interference during data forwarding [18]. In this case, it would be impossible for node $S$ to receive the accurate CTS and follow the correct path. In light of the non-interfering priority computation and the fixed number of related time slots, it is difficult to avoid traffic interference. What is more, with the increase in node density, the interference level rises up accordingly, as shown in Figure 3. 
Paper-Research on Interference Optimization Mechanism of Wireless Routing Signal Transmission..

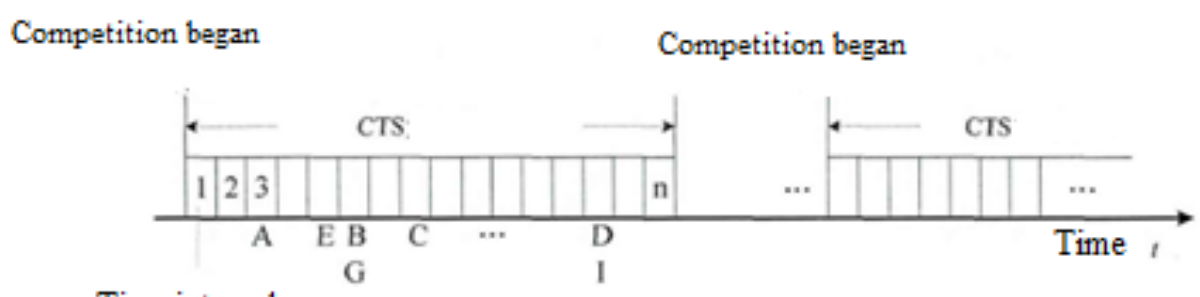

Time interval

a)

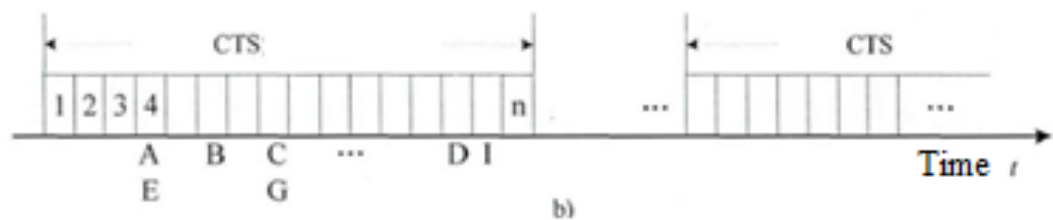

Fig. 2. Channel access based on competition windows

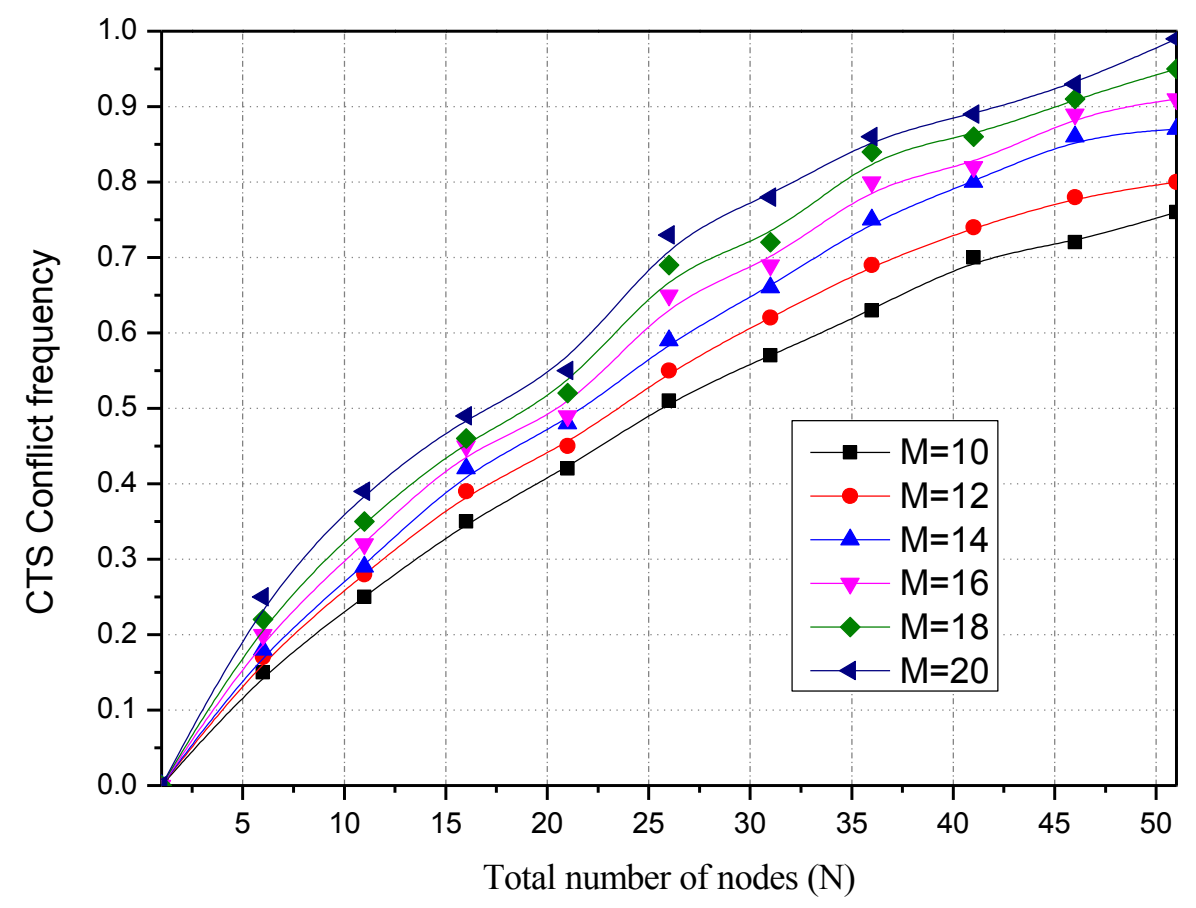

Fig. 3. CTS interference probability 


\section{Details of FCE design}

\subsection{Principles of FCE}

In order to solve the above problems, this paper proposes a mechanism of FCE. When FCE is used to allocate nodes, it is the number of candidate nodes rather than CTS competition windows that need to change, optimize and be selected. In this context, FCE mechanism provides an effective way to address the issue of traffic interference, selecting next-hop nodes of better performance. The fuzzy technology is the major foundation of node selection through FCE mechanism. Through the fuzzy comprehensive evaluation of the candidate node, those that are selected out will be more suitable to the next-hop competition. In this way, the CTS interference probability will be reduced effectively, and the forwarding efficiency will be improved remarkably [19]. In addition, this mechanism is also easy to adjust fuzzy evaluation parameters and the number of candidate nodes participating in the competition, thus effectively avoiding the influence of node density on node selection.

\subsection{The establishment of membership function}

Membership function should be established before nodes are selected through this mechanism. During the multi-hop forwarding, the relay node factor will significantly affect the performance of data transmission.

FCE mechanism determines the priority of candidate nodes based on influencing factors. The corresponding factor set as follows:

$\mathrm{U}=\{$ the expected forwarding distance of a node in one hop $(\mathrm{u} 1)$, node residual en$\operatorname{ergy}(\mathrm{u} 2)\}$

Equation (1) expresses the connotation of factor $\mathrm{u} 1$ and $\mathrm{u} 2$, where $\mathrm{F}$ and $\mathrm{C}$ denote the current node and the candidate node, respectively, and dist (F, Sin k) is the distance between the sending node and the base station. The analysis shows that the $u 1$ balances the one-hop forwarding distance, whose evaluation level may strongly impact on the reliability and sometimes the real-time property of information transmission. The same as the remaining energy, the value of the second factor can be used to evaluate the effect of nodes on network load.

$$
\left\{\begin{array}{l}
u_{1}=\left[\operatorname{dist}(F, \operatorname{Sin} k)-\operatorname{dist}(C, \operatorname{Sin} k) P_{C \cdot K}\right] \\
u_{2}=E_{\text {Remain }}
\end{array}\right.
$$

The comment expression of candidate nodes are as follows:

$V=\left\{\right.$ excellent $\left(v_{1}\right)$, intermediate $\left(v_{2}\right)$, poor $\left.\left(v_{3}\right)\right\}$

FCE mechanism designs corresponding membership function according to these factors, and defines the condition $\mathrm{U}=\left\{u_{1}, u_{2}\right\}$. What is more, each comment in the comment set $V=\left\{v_{1}, v_{2}, v_{3}\right\}$ is regarded as the fuzzy set of the corresponding discourse domain: $A_{v 1}$ excellent, $A_{v 2}$ intermediate, $A_{v 3}$ poor. In this way, factors can undergo fuzzy processing and evaluations, whose comments are $\gamma_{1}=\left\{A_{v 1}\left(u_{1}\right), A_{v 2}\left(u_{1}\right), A_{v 3}\left(u_{1}\right)\right\}$ 
and $\gamma_{2}=\left\{A_{v 1}\left(u_{2}\right), A_{v 2}\left(u_{2}\right), A_{v 3}\left(u_{2}\right)\right\}$. Factor membership function of the specific circumstances shown in Figure 4, the corresponding mathematical expression see (2).
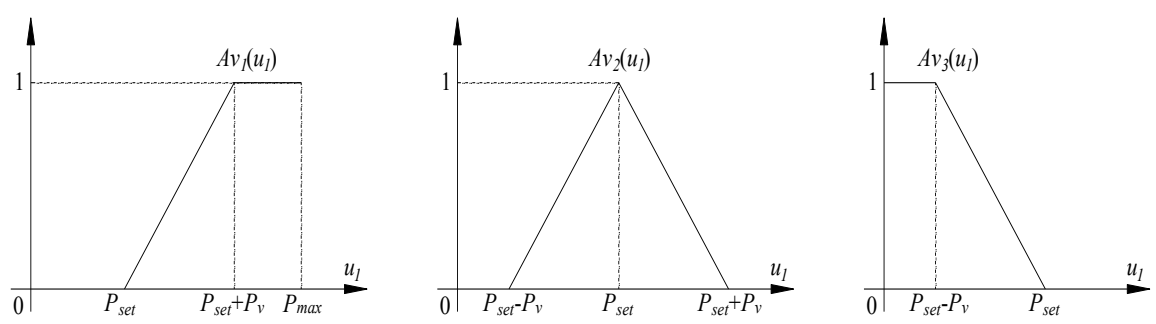

Fig. 4. The membership function of factor $u_{1}$

$$
\begin{aligned}
& A_{v 1}\left(u_{1}\right)=\left\{\begin{array}{l}
0 \quad u_{1} \leq P_{s e t} \\
\frac{u_{1}-P_{s e t}}{P_{v}} \quad P_{s e t} \leq u_{1} \leq\left(P_{s e t}+P_{v}\right) \\
1 \quad\left(P_{s e t}+P_{v}\right) \leq u_{1} \leq P_{\max }
\end{array}\right. \\
& A_{v 2}\left(u_{1}\right)= \begin{cases}0 & u_{1} \leq\left(P_{s e t}-P_{v}\right) \\
\frac{u_{1}-\left(P_{s e t}-P_{v}\right)}{P_{v}} & \left(P_{s e t}-P_{v}\right) \leq u_{1} \leq P_{s e t} \\
\frac{\left(\left(P_{s e t}+P_{v}\right)-u_{1}\right.}{P_{v}} & P_{s e t} \leq u_{1} \leq\left(P_{s e t}+P_{v}\right) \\
0 & \left(P_{s e t}+P_{v}\right) \leq u_{1} \leq P_{\max }\end{cases} \\
& A_{v 3}\left(u_{1}\right)=\left\{\begin{array}{l}
1 \quad u_{1} \leq\left(P_{s e t}-P_{v}\right) \\
\frac{P_{s e t}-u_{1}}{P_{v}} \quad\left(P_{s e t}-P_{v}\right) \leq u_{1} \leq P_{s e t} \\
0 \quad P_{s e t} \leq u_{1} \leq P_{\max }
\end{array}\right. \\
& P_{v}=\left\{\begin{array}{l}
\frac{\sqrt{3}}{3}\left(P_{\max }-P_{\text {set }}\right) \quad P_{\text {set }} \geq \frac{P_{\max }}{2} \\
\frac{\sqrt{3}}{3} P_{\text {set }} \quad P_{\text {set }} \leq \frac{P_{\max }}{2}
\end{array}\right.
\end{aligned}
$$

$P_{\max }$ represents the maximum theoretical value of factor $\mathrm{u} 1$ : yet we let it be the maximum communication radius of a node; $P_{\text {set }}$ represents the corresponding dynamic adjustment variable; the random variable will change with the change of $P_{\text {set. }}$. All nodes obey uniform distribution within a certain distance, but $\mathrm{Pv}$ is the mean square $\sqrt{D\left(u_{1}\right)}$ of the random variable $u_{1}$.

In this paper, we let $P_{\text {set }}$ be a "fuzzy evaluation parameter". According to the above discussion, the membership function that corresponds to the pair of factors can be 
determined by adjusting the value of $P_{\text {set }}$, and the membership evaluation results can be further determined accordingly.

\subsection{Fuzzy comprehensive evaluation method}

When the node is determined by the fuzzy comprehensive evaluation method, if it receives the BRTS from another node and then calculates to be qualified as the candidate node of the node, node A can undergo fuzzy comprehensive evaluation and identify the competition priority Pcts according to the evaluation result. Below is the corresponding procedures:

1. The candidate node A extracts the length information in the BRTS frame, and then determines the corresponding packet rate of the node according to Equation 1-3. On this basis, the value of factor $\mathrm{u} 1$ is determined: with which we calculate the corresponding residual energy of node $\mathrm{A}$ and determine the value of factor $\mathrm{u} 2$ accordingly.

2. The fuzzy evaluation parameter $P_{\text {set }}$ is then extracted from this frame and undertakes configuration of $A_{v l}\left(u_{i}\right), A_{v 2}\left(u_{i}\right), A_{v 3}\left(u_{i}\right)$ according to the $u_{i}(i=1,2)$ related membership function.

3. Afterwards, the single factor $\mathrm{u} 1$ is evaluated according to the membership function, and the corresponding evaluation set $\gamma_{i}$ can be determined by Equation 6:

$$
\gamma_{i}=\left(A_{v 1}\left(u_{i}\right), A_{v 2}\left(u_{i}\right), A_{v 3}\left(u_{i}\right)\right)
$$

On this basis, we define the node's comprehensive evaluation matrix, whose expression is shown in Equation 7

$$
R=\left\{\begin{array}{l}
A_{v 1}\left(u_{1}\right), A_{v 2}\left(u_{1}\right), A_{v 3}\left(u_{1}\right) \\
A_{v 1}\left(u_{2}\right), A_{v 2}\left(u_{2}\right), A_{v 3}\left(u_{2}\right)
\end{array}\right\}
$$

4. The fuzzy comprehensive evaluation set $\mathrm{Q}$ is determined by Equation 5, and the factor weight fuzzy subset $W=\left\{w_{1}, w_{2}\right\}$. The corresponding fuzzy evaluation model $M(\otimes, \oplus)$ and the influence of different factors on forwarding performance are determined by weighted average.

$$
Q=W \cdot R=\left\{\sum_{i=1}^{2} w_{i} \cdot A_{v 1}\left(u_{i}\right), \sum_{i=1}^{2} w_{i} \cdot A_{v 2}\left(u_{i}\right), \sum_{i=1}^{2} w_{i} \cdot A_{v 3}\left(u_{i}\right)\right\}=\left\{q_{1}, q_{2}, q_{3}\right\}
$$

5. According to the principle of maximum membership, there are mainly three class evaluation results in Q. If $j=1, q_{1}$ will be the highest class among them, which means it corresponds to class $\mathrm{v} 1$.

6. The node's priority can also be identified by equation 8 , in whose case the time of back-off timer should be preset. In this way, the class of candidate nodes can be determined. 


$$
P_{C T S}=\left\{\begin{array}{l}
\operatorname{Rand}\left(1, N_{v 1}\right) \cdot T_{\text {slot }} \quad(j=1) \mathrm{I} \quad\left(u_{1}>P_{\text {set }}\right) \\
N_{v 1}+\operatorname{Rand}\left(1, N_{v 2}\right) \cdot T_{\text {slot }} \quad(j=2) \mathrm{I} \quad(j=1) \mathrm{I} \quad\left(u_{1} \leq P_{\text {set }}\right) \\
N_{v 1}+N_{v 2}+\operatorname{Rand}\left(1, N_{v 3}\right) \cdot T_{\text {slot }} \quad j=3
\end{array}\right.
$$

Where $\operatorname{Rand}\left(1, N_{v l}\right)$ backs off to a random integer between 1 and $N_{v l}: T_{\text {slot }}$ is the length of a time slot; $N_{v 1}, N_{v 2}, N_{v 3}$ are the number of time slots, and satisfy equation 10.

$$
\left(N_{v 1}, \quad N_{v 2}, N_{v 3}\right) \cdot T_{\text {slot }} \leq \text { DIFS }
$$

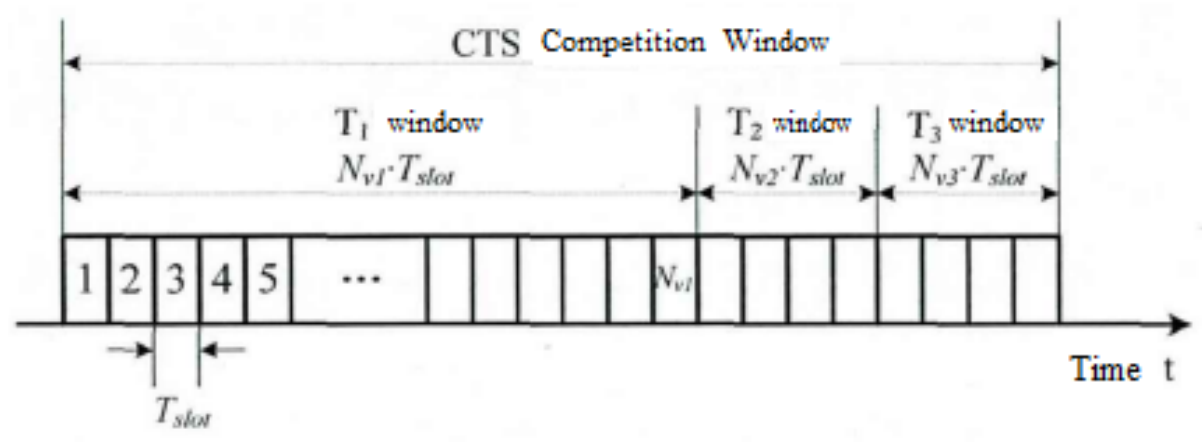

Fig. 5. Time slot distribution principle

Figure 5 is the specific flow of time slot distribution, in whose mechanism the CTS competition window is divided into three parts: $T_{1}, T_{2}, T_{3}$. Candidate nodes that meet the level requirements can be assigned corresponding time slots, some of which will be able to participate in the following competition of forwarding right. Meanwhile, the rest of the nodes will be assigned the time slots in window $T_{2}$ and window $T_{3}$. By node forwarding in $\mathrm{T} 2$ and $\mathrm{T} 3$, the monitoring work can be implemented, determining parameter setting.

\section{Simulation experiment and performance analysis}

\subsection{Simulation environment settings}

In this section, the FCE mechanism and OGF are realized in the simulation environment. Also, the corresponding route conflict probability is determined, with the comparative analysis of the transmission efficiency and time delay between different mechanisms. Details of parameter settings are listed in Table 1 and Table 2. 
Paper-Research on Interference Optimization Mechanism of Wireless Routing Signal Transmission..

Table 1. Parameter settings of the model

\begin{tabular}{|c|l|l|}
\hline Parameter & \multicolumn{1}{|c|}{ Instructions } & \multicolumn{1}{c|}{ Numerical } \\
\hline $\mathrm{B}_{\mathrm{N}}$ & Noise & $30 \mathrm{kHz}$ \\
\hline $\mathrm{R}$ & Rate & $19.2 \mathrm{kbps}$ \\
\hline $\mathrm{P}_{\mathrm{n}}$ & Noise base & $-115 \mathrm{dBm}$ \\
\hline $\mathrm{P}_{\mathrm{t}}$ & Send power & $0 \mathrm{dBm}$ \\
\hline $\mathrm{d}_{0}$ & Reference range & $1 \mathrm{~m}$ \\
\hline $\mathrm{N}$ & Loss & 10 \\
\hline $\mathrm{X}_{\mathrm{a}}$ & Standard deviation & 3 \\
\hline
\end{tabular}

Table 2. Parameter settings of energy consumption

\begin{tabular}{|l|c|c|}
\hline \multicolumn{1}{|c|}{ Running state } & Duration time(ms) & Working current $(\mathbf{m A})$ \\
\hline Seng 1byte $(0 \mathrm{dBm})$ & $0.416\left(\mathrm{~T}_{\mathrm{TX}}\right)$ & $20\left(\mathrm{I}_{\mathrm{TX}}\right)$ \\
\hline Receive 1byte & $0.416\left(\mathrm{~T}_{\mathrm{RX}}\right)$ & $15\left(\mathrm{I}_{\mathrm{RX}}\right)$ \\
\hline Monitor & $0.35\left(\mathrm{~T}_{\mathrm{CS}}\right)$ & $15\left(\mathrm{I}_{\mathrm{CX}}\right)$ \\
\hline
\end{tabular}

\subsection{Simulation result analysis}

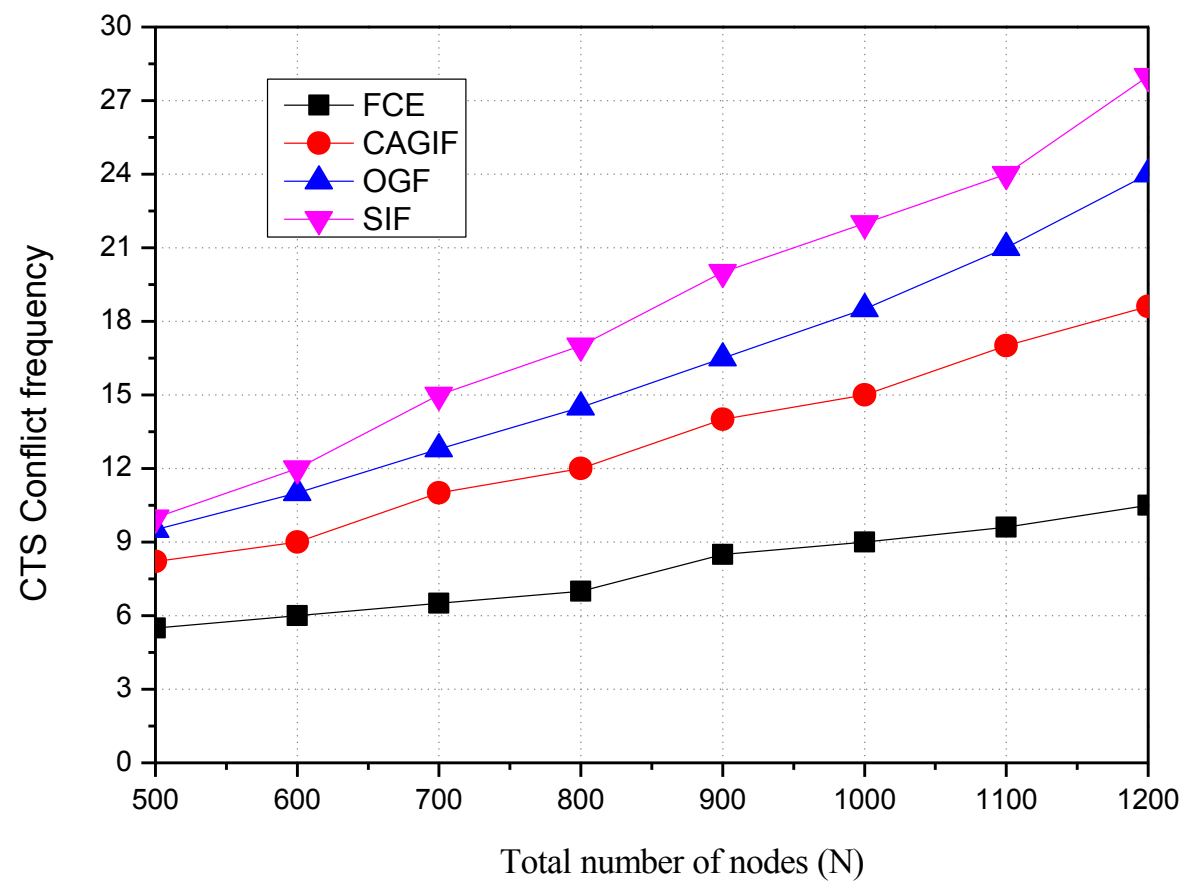

Fig. 6. The average interference times of CTS 
In this paper, we first test the number of route interferences of the four protocols. Figure 6 is the times of route interferences for each protocol to successfully transmit a data package network. The more the time is, the greater the interference is. According to the comparison results, the number of route interference under the other three protocols is much higher than that under the FCE protocol. What is more, as the nodes are distributed denser, the corresponding interference degree becomes higher. It can be seen from the comparison results that the CTS interference probability of the FCE mechanism is significantly smaller than others, and that the increment in interference degree is insignificant when the network nodes are denser. This is mainly because the advantages of distributed fuzzy comprehensive evaluation and those of priority competition are combined with each other, together contributing to the reduction in interference occurrence probability and to the improvement of routing efficiency.

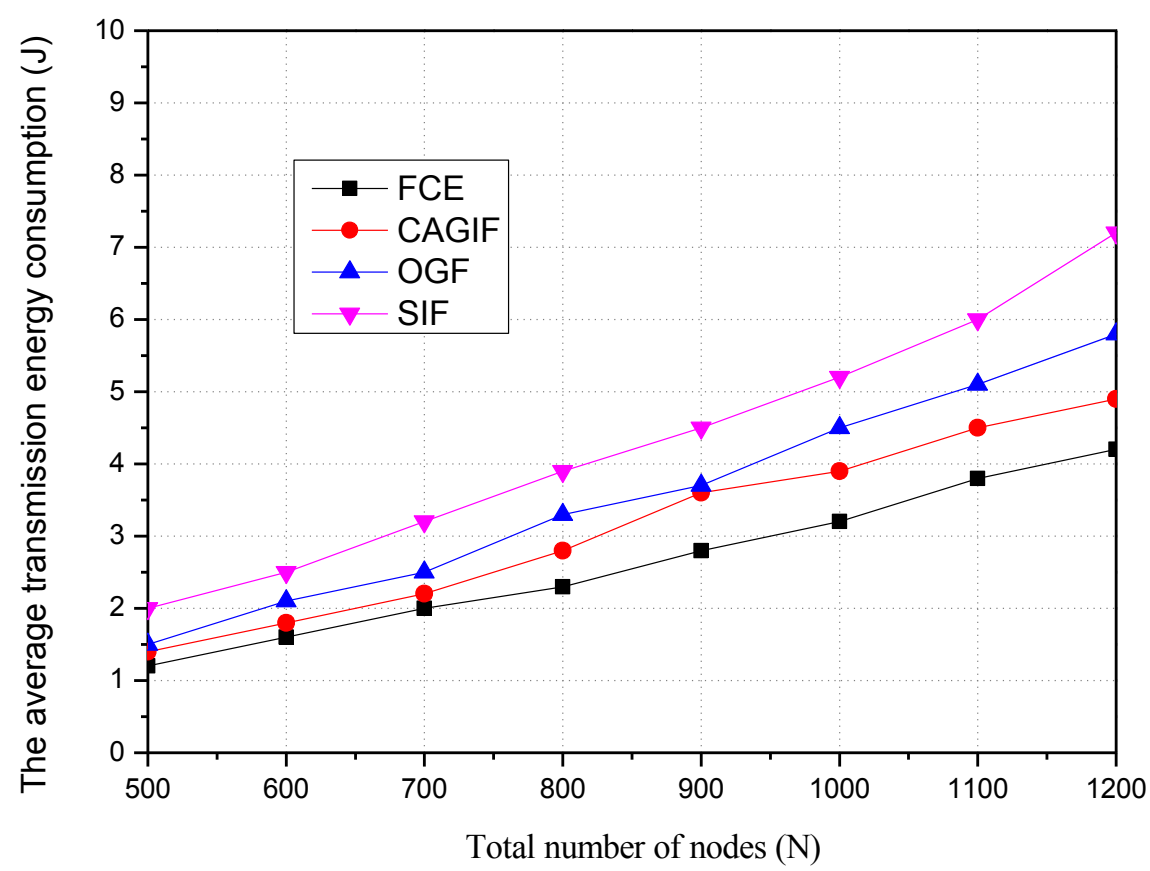

Fig. 7. Average transmission energy consumption

The average transmission efficiency of all of the protocols are tested in the condition that the transmission reliability is $94 \%$, when energy consumption is negatively correlated with transmission efficiency. The specific results are shown in Figure 7. Through analysis of the figure, it can be seen that when the network nodes are not densely distributed, the difference of transmission-related energy consumption is insignificant for different protocols; as node density increases, the energy consumption of all protocols but FCE protocol rockets, while FCE protocol rises slowly and remains at the lowest level among other protocols'. SIF and OGF have the respective highest and the second highest levels of energy consumption. 
By analyzing Figure 6-7, it can be found that when the network node density is large, the interference level of every protocol maintains at a high level, which incurs larger competition-related energy consumption. Such being the case, FCE protocol effectively optimizes routing competition, providing an efficacious way to reduce energy consumption.

\section{Conclusion}

This paper is largely aimed at solving the problem of routing interference, for which it proposes a route interference optimization mechanism FCE based on distributed fuzzy comprehensive evaluation. As to this mechanism, the fuzzy comprehensive evaluation technique is used to select candidate nodes, with the advantage of node selection optimization by balancing node forwarding distance, communication quality and other related factors. In this way, great reduction is achieved in the number of candidate nodes, route interference and energy consumption. The way to select nodes under this mechanism is to endow priorities for multiple candidate nodes and to choose nodes of better comprehensive performance from the candidate nodes according to some influencing factors. In this way, not only can the competition-induced route interference is reduced, but the energy consumed during node competition becomes less. The computational load of this mechanism is not great, as all candidate nodes are processed through fuzzy comprehensive evaluation distribution. The low demand for computational ability also helps increase the scalability of protocols.

\section{References}

[1] Muruganathan, S. D., Ma, D. C. F., Bhasin, R. I., Fapojuwo, A. (2005). A centralized energy-efficient routing protocol for wireless sensor networks. IEEE Communications Magazine, 43(3), S8-13. https://doi.org/10.1109/MCOM.2005.1404592

[2] Chen, G., Li, C., Ye, M., Wu, J. (2009). An unequal cluster-based routing protocol in wireless sensor networks. Wireless Networks, 15(2), 193-207. https://doi.org/10.1007/s11276007-0035-8

[3] Nasser, N., Chen, Y. (2007). Seem: secure and energy-efficient multipath routing protocol for wireless sensor networks. Computer Communications, 30(11-12), 2401-2412. https://doi.org/10.1016/j.comcom.2007.04.014

[4] Tsai, M. J., Yang, H. Y., Liu, B. H., Huang, W. Q. (2009). Virtual-coordinate-based delivery-guaranteed routing protocol in wireless sensor networks. IEEE/ACM Transactions on Networking, 17(4), 1228-1241. https://doi.org/10.1109/TNET.2008.2008002

[5] Liu, M., Cao, J., Chen, G., Wang, X. (2009). An energy-aware routing protocol in wireless sensor networks. Sensors, 9(1), 445-462. https://doi.org/10.3390/s90100445

[6] Lu, Y. M., Wong, V. W. S. (2006). An energy-efficient multipath routing protocol for wireless sensor networks. International Journal of Communication Systems, 20(7), 1-5. https://doi.org/10.1109/vtcf.2006.505

[7] Ahmed, A. A., Fisal, N. (2008). A real-time routing protocol with load distribution in wireless sensor networks. Computer Communications, 31(14), 3190-3203. https://doi.org/10.1016/j.comcom.2008.04.030 
Paper-Research on Interference Optimization Mechanism of Wireless Routing Signal Transmission...

[8] Sanchez, J. A., Ruiz, P. M., Liu, J., Stojmenovic, I. (2007). Bandwidth-efficient geographic multicast routing protocol for wireless sensor networks. IEEE Sensors Journal, 7(5), 627-636. https://doi.org/10.1109/JSEN.2007.894149

[9] Yu, J., Qi, Y., Wang, G., Gu, X. (2012). A cluster-based routing protocol for wireless sensor networks with nonuniform node distribution. AEU - International Journal of Electronics and Communications, 66(1), 54-61. https://doi.org/10.1016/j.aeue.2011.05.002

[10] Chiang, S. S., Huang, C. H., Chang, K. C. (2007). A minimum hop routing protocol for home security systems using wireless sensor networks. IEEE Transactions on Consumer Electronics, 53(4), 1483-1489. https://doi.org/10.1109/TCE.2007.4429241

[11] Wei, C., Zhi, C., Fan, P., Letaief, K. B. (2009). Asor: an energy efficient multi-hop opportunistic routing protocol for wireless sensor networks over rayleigh fading channels. IEEE Transactions on Wireless Communications, 8(5), 2452-2463. https://doi.org/10.1109/ TWC.2009.071388

[12] Zabin, F., Misra, S., Woungang, I., Rashvand, H. F., Ma, N. W., Ali, M. A. (2008). Reep: data-centric, energy-efficient and reliable routing protocol for wireless sensor networks. Iet Communications, 2(8), 995-1008. https://doi.org/10.1049/iet-com:20070424

[13] Tunca, C., Isik, S., Donmez, M. Y., Ersoy, C. (2015). Ring routing: an energy-efficient routing protocol for wireless sensor networks with a mobile sink. IEEE Transactions on Mobile Computing, 14(9), 1947-1960. https://doi.org/10.1109/TMC.2014.2366776

[14] Shi, L., Zhang, B., Mouftah, H. T., Ma, J. (2011). Ddrp: an efficient data-driven routing protocol for wireless sensor networks with mobile sinks. International Journal of Communication Systems, 26(10), 1341-1355.

[15] Jin, R. C., Gao, T., Song, J. Y., Zou, J. Y., Wang, L. D. (2013). Passive cluster-based multipath routing protocol for wireless sensor networks. Wireless Networks, 19(8), 1851-1866. https://doi.org/10.1007/s11276-013-0570-4

[16] Alrajeh, N. A., Khan, S., Lloret, J., Loo, J. (2013). Secure routing protocol using crosslayer design and energy harvesting in wireless sensor networks. International Journal of Distributed Sensor Networks, 2013(1), 893-900. https://doi.org/10.1155/2013/374796

[17] Xia, L., Chen, X., Guan, X. (2005). A new gradient-based routing protocol in wireless sensor networks. Lecture Notes in Computer Science, 5(4), 318-325. https://doi.org/10.1007/11535409 45

[18] Abdul, W., Sungwon, L., Dongkyun, K. (2012). A reliable and energy-efficient routing protocol for underwater wireless sensor networks. International Journal of Communication Systems, 27(10), 2048-2062.

[19] Jung, S. G., Kang, B., Yeoum, S., Choo, H. (2016). Trail-using ant behavior based energyefficient routing protocol in wireless sensor networks. International Journal of Distributed Sensor Networks, 2016(4), 1-9. https://doi.org/10.1155/2016/7350427

\section{$7 \quad$ Authors}

Xinchun Wang (corresponding author) is with College of Science, North China University of Science and Technology, Tangshan 063009, China. She was born in Hebei, China, in 1977. She works in School of North China University of Science and Technology, and received the Master's degree in 2010 from North China University of Science and Technology. Her field of interest is operation and optimization (10759697@qq.com). 
Haishan Zhang is with Modern Technology and Education Centre, North China University of Science and Technology, Tangshan 063009, China. He was born in Hebei, China, in 1981. He works in School of North China University of Science and Technology, and received the Master's degree in 2011 from North China University of Science and Technology. His field of interest is wireless sensor networks, image processing(38775617@qq.com).

Mi Li is with College of Life Science, North China University of Science and Technology, Tangshan 063009, China. She was born in Hebei, China, in 1987. She works in School of North China University of Science and Technology, and received the Master's degree in 2012 from North China University of Science and Technology. Her field of interest is operation and optimization (994177231@qq.com).

Ying Li is with College of Science, North China University of Science and Technology, Tangshan 063009, China. She was born in Hebei, China, in 1979. She works in School of North China University of Science and Technology, and received the Master's degree in 2011 from North China University of Science and Technology. Her field of interest is operation and optimization (1965063794@qq.com).

Article submitted 02 February 2017. Published as resubmitted by the authors 15 March 2017. 